\title{
FLUCTUATION OF THE NUCLEOTIDE POOLS OF FLAVINOGENIC AND NONFLAVINOGENIC STRAINS OF EREMOTHECIUM ASHBYII GROWN IN THE PRESENCE OF PURINES ${ }^{1}$
}

\author{
Hisateru Mitsuda, Yuzuru SuzuKI, and Kenji NAKAJIMA ${ }^{2}$ \\ Laboratory of Nutritional Chemistry, Faculty of Agriculture, \\ Kyoto University, Sakyo-ku, Kyoto 606 \\ (Received November 9, 1972)
}

Fluctuation of the nucleotide pools in a flavinogenic and a nonflavinogenic strains of Eremothecium ashbyii was followed by using column chromatography with an anion exchanger, and effects of purine bases on the pools were tested throughout the growth cycle.

1) Contents of the nucleotides in the flavinogenic strain were detected to be maximum at 1 day culture prior to the growth maximum ( 2 days culture), while in the nonflavinogenic strain the largest pools were observed after 4 days culture, corresponded to the growth maximum. After 1 day cultivation the pools in the former strain shrank rapidly with the culture time: this was remarkable for ATP pool.

2) It was found in the flavinogenic fungus that GMP pool decreased more slowly than AMP pool at the exponential stage of riboflavin formation.

3) Addition of purine bases into the culture media resulted in expantion of ATP pool and simultaneous shrink of AMP pool in the flavinogenic strain, although sum of the contents of adenosine nucleotides remained almost constant. However, such phenomenon was not observed in the nonflavinogenic strain.

4) Growth maximum of the nonflavinogenic fungus was obtained at 4 days culture, but riboflavin was synthesized with the maximum yield after 7 days cultivation.

It is probable that there is an intimate relationship between riboflavin production and fluctuation of nucleotide pools in flavinogenic microbes, because purine

\footnotetext{
1 The contents of this paper were partially presented at the 205th Vitamin B Research Committee (October 1971).

2 満田久輝, 鈴木 譲, 中島謙二
} 
bases can be synthesized de novo in their nucleotide forms $(1)$ and also incorporated without degradation into the skeleton of the vitamin(2-4). The dynamic aspects of the relationship, however, have never been explored in detail. In this work with a flavinogenic and nonflavinogenic fungi, both strains of Eremothecium ashbyii, an attempt was made to elucidate the correlation of vitamin synthesis with changes in the nucleotide pools during fungal development and the effect of exogenous purine bases on the pools.

\section{EXPERIMENTAL}

Materials. Inosine diphosphate (IDP), nicotineamide adenine dinucleotide (NAD), nicotineamide adenine dinucleotide phosphate (NADP), flavin adenine dinucleotide (FAD), guanosine diphosphate (GDP), guanosine triphosphate (GTP), cytidine monophosphate (CMP), and cytidine triphosphate (CTP) were purchased from Sigma Company; riboflavin, adenine, guanine, hypoxanthine, xanthine, and adenosine monophosphate (AMP) from Nakarai Chemicals, Ltd., Kyoto; inosine monophosphate (IMP), guanosine monophosphate (GMP), and urdine monophosphate (UMP) from Takeda Chemical Industries, Ltd.; and analytical grade Dowex $1 \times 2\left(\mathrm{Cl}^{-}\right)(200-400 \mathrm{mesh})$ from Dow Chemicals. Guanosine diphosphate mannose (GDP-M), uridine diphosphate galactose (UDP-Gal), and uridine diphosphate glucose (UDP-G) were obtained through the courtesy of Prof. K. Ogata, Department of Agriculture, Kyoto University. All other chemicals used were of the best quality available.

Organisms. E. ashbyii strain IFO 0944 (flavinogenic) and IFO 1425 (nonflavinogenic) were obtained through the courtesy of the Institute for Fermentation, Osaka. The molds were maintained as described previously(5).

Fungal growth and determination of riboflavin. The basal medium ( $\mathrm{pH} 5.5)$ contained $2 \%$ glucose, $1.8 \%$ Tween $80,1 \%$ peptone, $0.3 \%$ yeast extract, $0.2 \%$ $\mathrm{KH}_{2} \mathrm{PO}_{4}, 0.1 \% \mathrm{NaCl}, 0.01 \% \mathrm{MgSO}_{4} 7 \mathrm{H}_{2} \mathrm{O}$, and distilled water. Experimental procedures for fungal growth and determination of riboflavin production employed were described previously(5).

Extraction of Nucleotides and Column Chromatography. At the end of the defined period of the fermentation, which was carried out in the basal medium (500 $\mathrm{ml}$ in 2-liter Erlenmeyer flask) under similar conditions to those described previously(5), the mycelia were harvested through filter paper under suction, followed by washing with chilled distilled water. The mycelia $(10 \mathrm{~g})$ were mixed immediately with distilled water $\left(20 \mathrm{ml}, 4^{\circ} \mathrm{C}\right)$, and then $3 \mathrm{M} \mathrm{HClO}_{4}\left(4 \mathrm{ml}, 4^{\circ} \mathrm{C}\right)$ was added to the mixtures. Hand grinding in a mortar was continued for $3 \mathrm{~min}$ in the cold. After the suspension was centrifuged at $0^{\circ} \mathrm{C}$, the extract was neutralized by $3 \mathrm{M} \mathrm{KOH}\left(4^{\circ} \mathrm{C}\right)$. The resulting $\mathrm{KClO}_{4}$ was removed by decantation. The supernatant $(10 \mathrm{ml})$ was applied to a Dowex $1 \times 2$ (formate) column $(0.7 \times 30 \mathrm{~cm})$ prepared by a method similar to that of HULBERT et al.(6). The column was washed 
with distilled water $(40 \mathrm{ml})$, and the washing speed was adjusted to $2 \mathrm{ml}$ per $5 \mathrm{~min}$. The column was developed by a HCOOH-HCOONH${ }_{4}$ elution system(6) with a mixing chamber of distilled water $(50 \mathrm{ml})$ and a reservoir containing in order one of the following solvents: solvent I, $0.5 \mathrm{M}$ formic acid $(100 \mathrm{ml})$; solvent II, $2 \mathrm{M}$ formic acid $(100 \mathrm{ml})$; solvent $\mathrm{III}, 2.5 \mathrm{M}$ formic acid- $0.18 \mathrm{M}$ ammonium formate $(100 \mathrm{ml})$; solvent IV, $3 \mathrm{M}$ formic acid- $0.31 \mathrm{M}$ ammonium formate $(150 \mathrm{ml})$; and solvent V, $3 \mathrm{M}$ formic acid-2.2 $\mathrm{M}$ ammonium formate $(100 \mathrm{ml})$.

Charcoal Adsorption. The nucleotides in the column effluents were adsorbed on acid-washed charcoal to separate them from formate and ammonium formate. The charcoal was collected by filtration through filter paper, followed by washing with distilled water. The nucleotides were eluted from the charcoal by a mixture of $5 \%$ ammonia water in $60 \%$ ethanol.

The eluate was taken to dryness under reduced pressure. Samples prepared by this procedure were submitted to nucleotide identification.

Paper chromatography. The following solvent systems were used for paper chromatography of various nucleotides: solvent 1 , acetone- $25 \%$ trichloroacetic acid $(75: 25)(7)$, solvent $2,0.1 \mathrm{M}$ phosphate buffer at $\mathrm{pH} 6.8$-ammonium sulfate$n$-propanol (100 ml: $60 \mathrm{~g}: 2 \mathrm{ml})(8)$; solvent 3, isopropanol-1 $\mathrm{N}$ ammonia solution $(70: 30)(9)$; solvent 4 , collidine saturated by water(10); solvent $5,1 \mathrm{M}$ acetate buffer at $\mathrm{pH} 3.8$-ethanol $(3: 7.5)(11)$; and solvent $6, n$-butanol-acetic acid-water $(4: 1$ : 1)(12). The chromatograms were developed by the ascending method at room temperature. Sugar nucleotides could be separated from each other chromatographically by solvents 2 and 5. Paper chromatography for sugar compounds was carried out in solvent systems of $n$-butanol-pyridine-water $(3: 2: 1.5)$ (solvent 7)(13) and of ethyl acetate-water-pyridine $(2: 2: 1)$ (solvent 8$)(14)$. The nucleotides were formed as dark spots and riboflavin derivatives as yellow fluorescent spots under ultraviolet light. Sugar compounds were identified by spraying the chromatograms with alkaline silver solution(15) or with benzidine-trichloroacetic acid mixture(12).

Acid hydrolysis. In order to identify the base components of nucleotides, hydrolysis of the nucleotides was carried out by heating at $100^{\circ} \mathrm{C}$ for $1 \mathrm{hr}$ in $1 \mathrm{~N}$ $\mathrm{HCl}(16)$. The resulting solution was evaporated, and the dried matter was examined by paper chromatography. A mild hydrolysis of sugar nucleotides by heating at $100^{\circ} \mathrm{C}$ for 10 to $15 \mathrm{~min}$ in $0.005 \mathrm{~N} \mathrm{HCl}$ was employed to determine the sugar moieties of the nucleotides(11). The mixtures were dried under vacuum. The dried matter was dissolved in distilled water and applied to a Dowex $50 \mathrm{~W} \times 4-(\mathrm{H}+)$ column and an Amberlite IR-4B column. The column effluent was evaporated and examined by chromatography.

Determination of Nucleotides. Identification of nucleotides from the column chromatography was accompanied by: (a) paper chromatography together with known samples; (b) comparing elution behavior during the column chromatography with known samples; and (c) measuring ultraviolet absorption spectra of the isolated 
compounds. These methods were also used with the products from the hydrolysis experiments in $\mathrm{HCl}$.

\section{RESULTS}

1. General patterns of nucleotide pools with growth of a flavinogenic strain of $E$. ashbyii

The three chromatograms in Fig. 1 show typical profiles of column chromatography of the acid soluble fractions from a flavinogenic strain of E. ashbyii. The 1-day cultured mycelia (log phase) contained more nucleotides than the 3- and 6-day cultured mycelia did. Especially compared with other nucleotides, nucleotides such as AMP, ATP, ADP, and UDP-gal showed higher contents. Conversely, fractions of riboflavin derivatives were observed in only small amounts. Flavin mononucleotide (FMN) could not be detected. In the mycelia after 3 days fermentation, at which time autolysis of the cell and riboflavin formation had begun, a marked decrease in the nucleotide pools resulted. Such a tendency was especially marked with ATP and GTP pools. Simultaneously, free flavin and FAD were accumulated in large amounts within cells. After 6 days of fermentation, at which time riboflavin formation attained the maximum yields, there was a drastic reduction in the nucleotide contents but a large increase of free vitamin and of FAD pools. It is to be noted that the NAD pool showed only a moderate decrease throughout the life cycle.

The relationship between the nucleotide pools and the life cycle is given in Table 1 for adenosine nucleotides and NAD. An extreme drop in the ATP pool

Table 1. Change of relative amounts of adenosine nucleotides with growth stage of $E$. ashbyii.

The data were calculated from the results of Fig. 1. Only the quantitative ratios concerning adenosine nucleotides are shown.

\begin{tabular}{lcccccc}
\hline & \multicolumn{5}{c}{ Cultivation time } \\
\cline { 2 - 7 } & \multicolumn{2}{c}{1 day } & \multicolumn{2}{c}{3 days } & 6 days \\
\hline NAD & 1 & - & 1 & - & 1 & - \\
& $\left(0.37^{\mathrm{a}}\right)$ & & $\left(0.29^{\mathrm{a}}\right)$ & & $\left(0.18^{\mathrm{a}}\right)$ & \\
AMP & 2.4 & 1 & 1.6 & 1 & 1.2 & 1 \\
ADP & 1.8 & 0.78 & 0.91 & 0.55 & 0.66 & 0.56 \\
ATP & 2.2 & 0.95 & 0.58 & 0.35 & 0.35 & 0.30 \\
AMP +ADP +ATP & 6.4 & - & 3.1 & - & 2.2 & \\
\hline
\end{tabular}

a $\mu$ moles as AMP.

Fig. 1. Change of nucleotide pool of E. ashbyii in the course of growth. Acid soluble extract $(10 \mathrm{ml})$ was applied on a column of Dowex $1 \times 2$ (formate $^{-}$) and separated by stepwise gradient elution. Flow rate was $0.9 \mathrm{ml}$ per $5 \mathrm{~min}$. Fractions of $1.8 \mathrm{ml}$ were collected. The absorbancy of eluate was automatically recorded by Uvicord. For details of chromatography see Experimentals. 


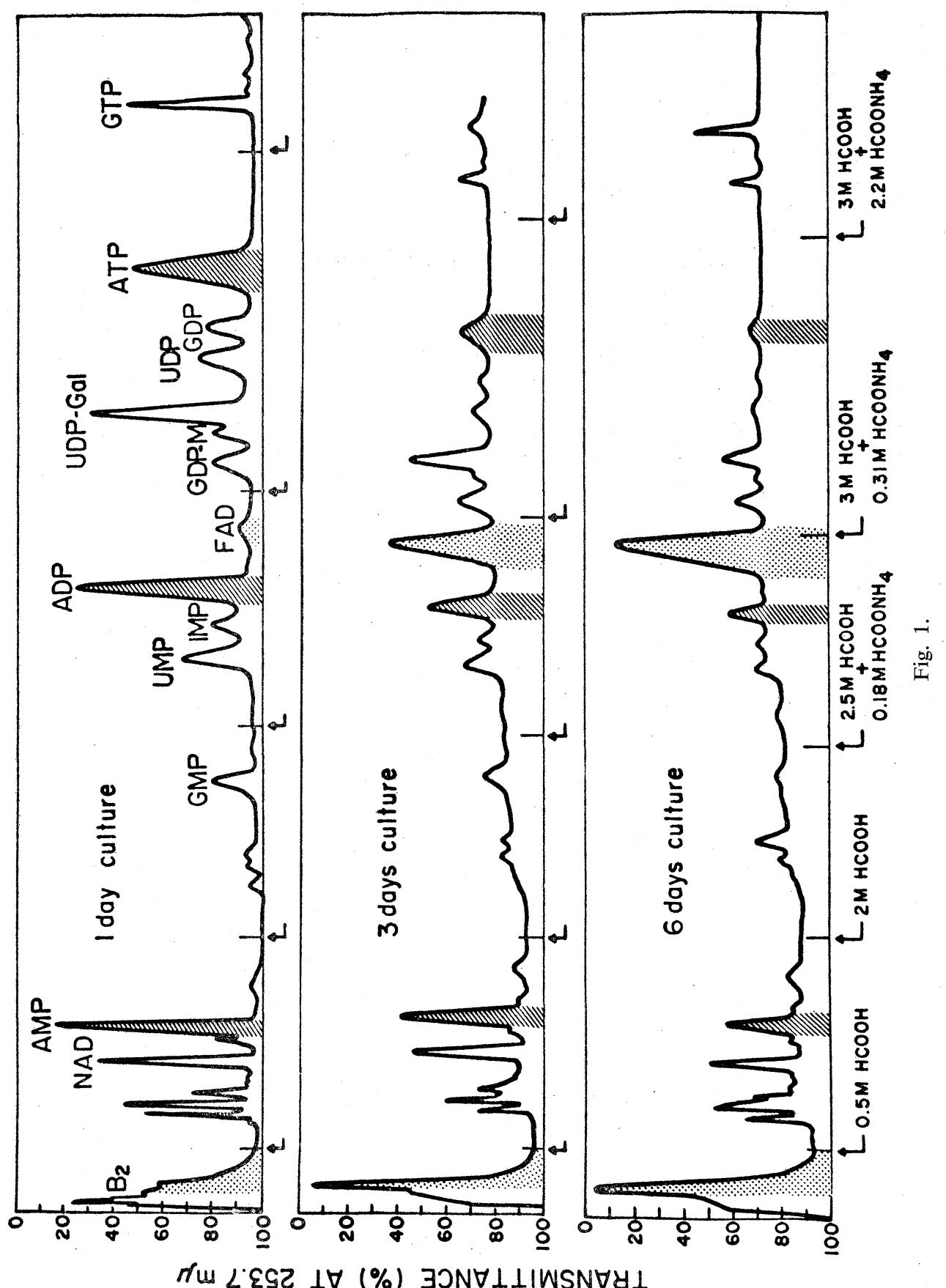


was brought about after the maximum growth, and the pool was drained off at the last stage of the life cycle. There was a change in ratio among AMP, ADP and ATP pools, the ratio of ATP/AMP reducing with growth.

Using rough calculations from data shown in Fig. 1 and other experiments not reported in this paper, the sum of the AMP, GMP, and IMP found on the chromatogram and the ratio of GMP/AMP are plotted against culture days as seen in Fig. 2.

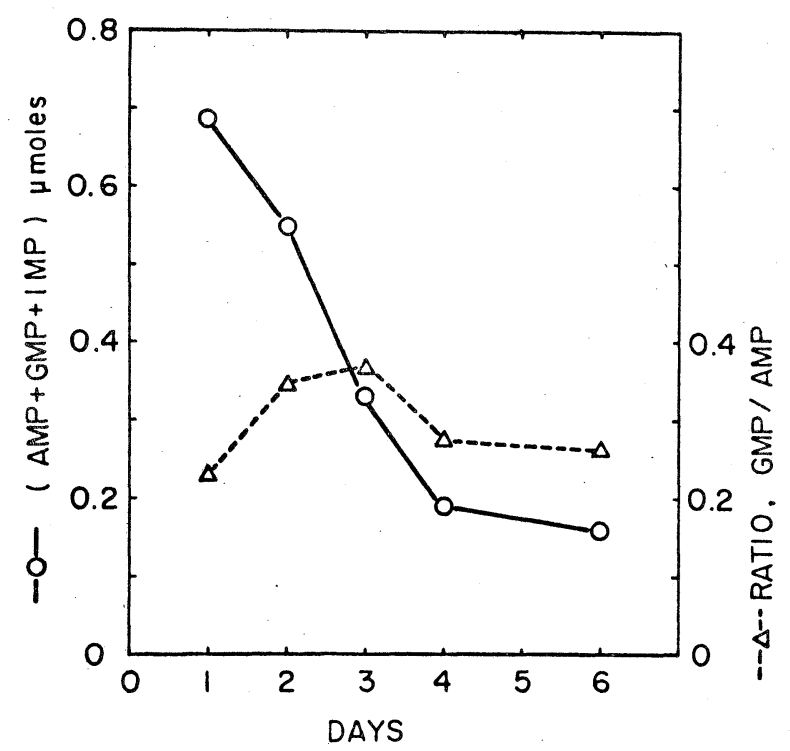

Fig. 2. Decrease of purine nucleotide monophosphates and the ratio of GMP/AMP during the culture of $E$. ashbyii. The relative amounts of purine nucleotide monophosphates were calculated from the results of Fig. 1 and results not described in this paper.

These data indicate that the maximum amounts of purine nucleoside monophosphates are observed at 1 day, corresponding to the logarithmic phase of growth, and thereafter they rapidly decrease linearly until 4 days, at which time the mycelia are almost completely autolyzed but the riboflavin content shows values near the maximum yeilds (17).

On the other hand, the values of GMP/AMP were maximum at 3 days, which corresponded to the logarithmic phase of riboflavin production, unlike the sum of purine nucleoside monophosphates. After 4 days of cultivation near the cessation of riboflavin formation the ratio decreased almost to the starting values of 1 day. This indicates different rates of reduction of GMP and AMP with flavinogenesis and also indicates that, especially in the exponential phase of riboflavin formation, the reduction of the GMP pool proceeds at a slower rate than that of the AMP pool. Accordingly, the linear reduction of AMP, GMP, and IMP 


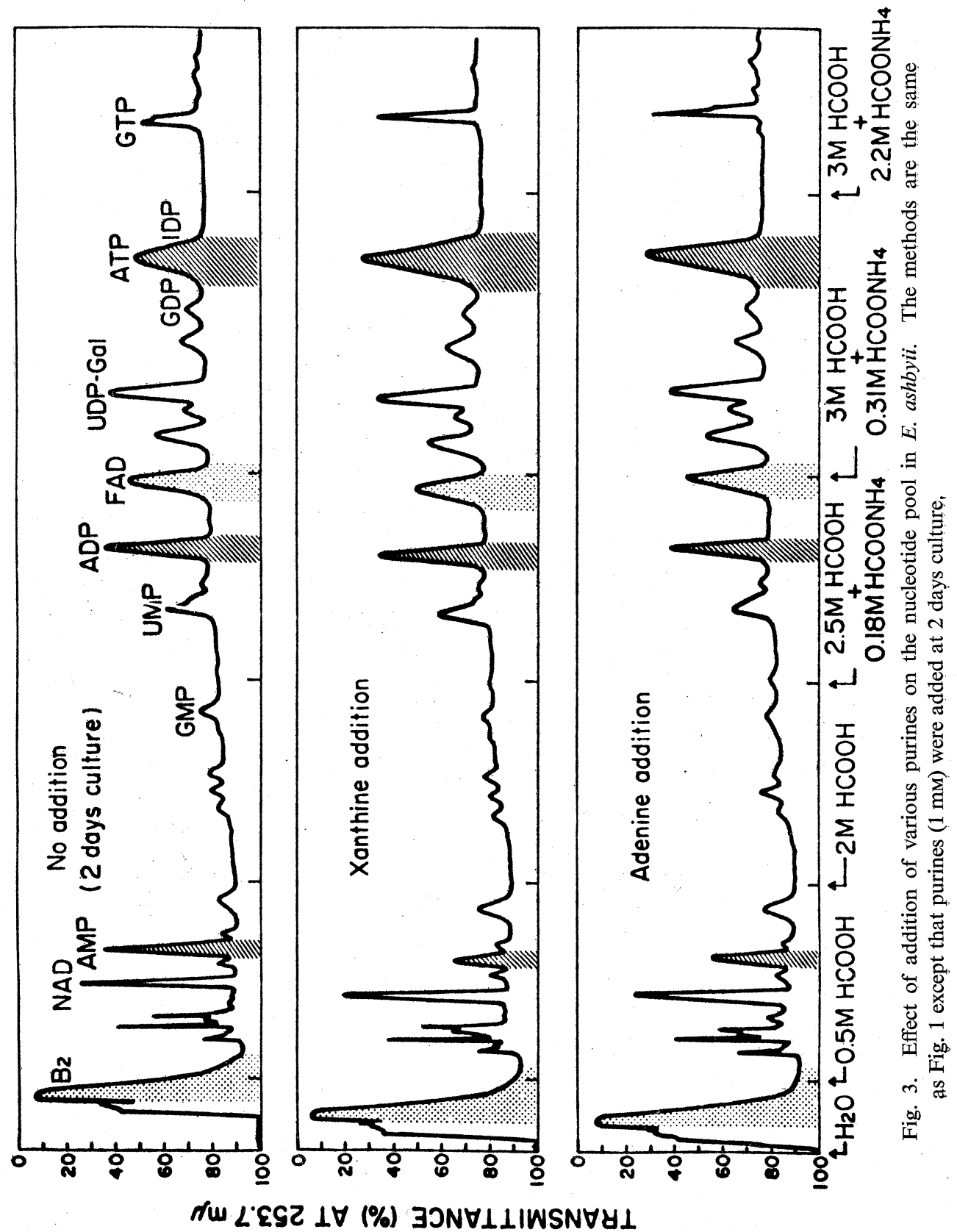


pools after the maximum growth and especially the preservation of the GMP pool at the logarithmic phase of riboflavin formation seems to be closely related to flavinogenesis.

2. Effects of purines on the nucleotide pools in a flavinogenic strain of E. ashbyii

Figure 3 shows the effects of the addition of adenine and xanthine on the nucleotide compositions in 2-day cultured mycelia of a flavinogenic strain kept at maximum growth rate. As shown in this figure, the addition elicited a marked increase in ATP level. Conspicuous influence of the addition, however, was not observed in the general chromatographic patterns of other nucleotides except ATP and AMP. This phenomenon was also induced to varied extents by other purines such as guanine and hypoxanthine. These relations are quantitatively represented in Table 2, in which the concentrations of nucleotides are expressed as relative values against those of NAD. The total amounts of adenosine nucleotides did not undergo an extreme change. However, the ratio of AMP, ADP and ATP was found to be altered. For all purines added the values of ATP/AMP increased. Xanthine was the most effective among the purines in increasing the ratio, in which the amount of ATP was about tenfold the amount of AMP. This increasing effect by purines were observed in the decreasing order of xanthine, guanine, adenine, and hypoxanthine. In the case of hypoxanthine, the ratio was shown to be only 2 times.

Table 2. Effect of purines on relative amounts of adenosine nucleotides in E. ashbyii.

The relative amounts were calculated from Fig. 3 and data not described in this paper.

\begin{tabular}{|c|c|c|c|c|c|c|c|c|c|c|}
\hline \multirow[b]{3}{*}{ NAD } & \multicolumn{10}{|c|}{ Addition } \\
\hline & \multicolumn{2}{|c|}{ None } & \multicolumn{2}{|c|}{ Xanthine } & \multicolumn{2}{|c|}{ Adenine } & \multicolumn{2}{|c|}{ Guanine } & \multicolumn{2}{|c|}{ Hypoxanthine } \\
\hline & $\begin{array}{c}1 \\
\left(0.28^{a}\right)\end{array}$ & - & $\begin{array}{l}1 \\
\left(0.47^{a}\right)\end{array}$ & - & $\stackrel{1}{\left(0.48^{a}\right)}$ & - & $\begin{array}{l}1 \\
\left(0.48^{a}\right)\end{array}$ & - & $\begin{array}{l}1 \\
\left(0.45^{a}\right)\end{array}$ & - \\
\hline AMP & 1.3 & 1 & 0.27 & 1 & 0.46 & 1 & 0.45 & 1 & 0.95 & 1 \\
\hline ADP & 1.5 & 1.2 & 0.66 & 2.5 & 0.93 & 2.0 & 1.0 & 2.3 & 1.0 & 1.1 \\
\hline ATP & 1.6 & 1.3 & 2.7 & 10.0 & 2.1 & 4.5 & 2.1 & 4.6 & 2.1 & 2.1 \\
\hline$\underset{\mathrm{ATP}}{\mathrm{AMP}+\mathrm{ADP}+}$ & 4.4 & - & 3.6 & 一 & 3.5 & - & 3.6 & - & 4.1 & - \\
\hline
\end{tabular}

a $\mu$ moles as AMP.

3. Changes of nucleotide pools with growth of a nonflavinogenic strain of E. ashbyii and effects of purines on these pools

The fluctuation of the nucleotide pools was studied in the nonflavinogenic strain in the same way as in the flavinogenic strain. However, the fermentational features of the nonflavinogenic strain are distinguishable from the flavinogenic strain even under the same cultural conditions. Figure 4 demonstrates the 
general patterns of the culture of the nonflavinogenic strain in a natural nutrient medium containing glucose, peptone, yeast extract, salts, and Tween 80 .

Growth achieved the maximum point at 4 days unlike the 2 days for the flavinogenic strain, and riboflavin formation showed the maximum yields at 7 days. However, the yields exhibited remarkably low values compared to the

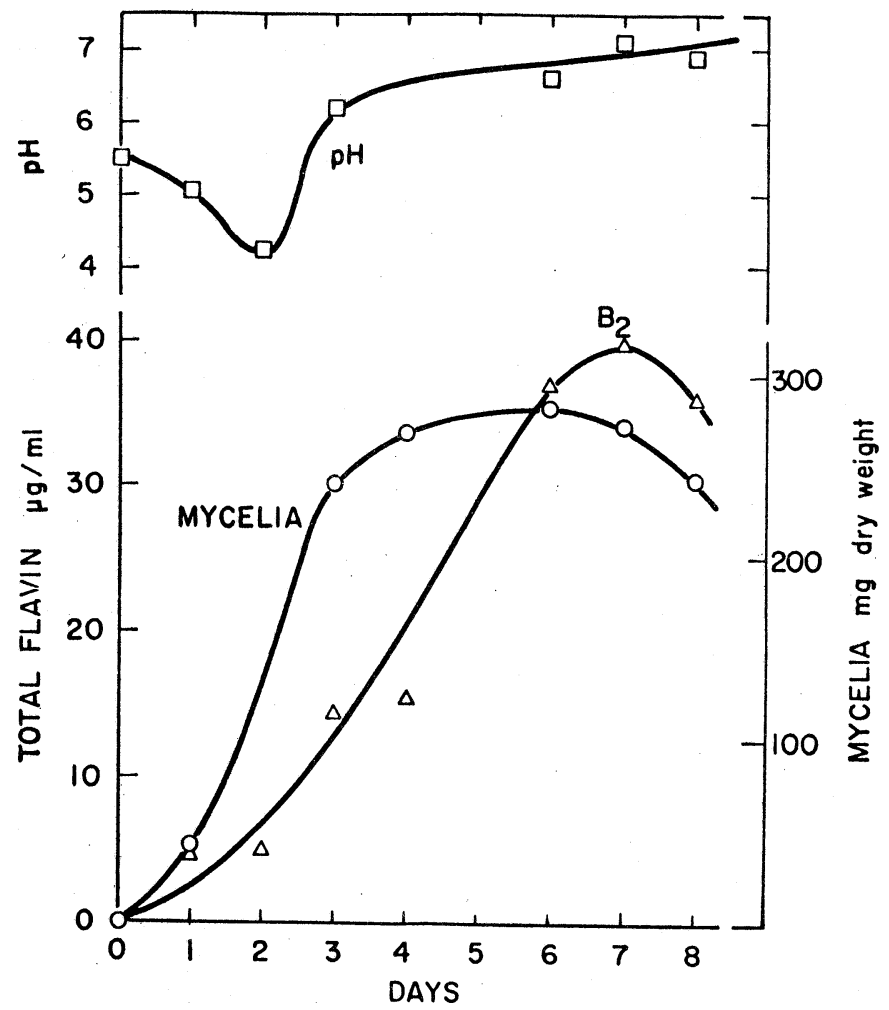

Fig. 4. Riboflavin synthesis by nonflavinogenic E. ashbyii on a basal medium. Mycelia were cultivated at $27^{\circ} \mathrm{C}$ by rotary shaker. Basal medium consisted of $2 \%$ glucose, $1 \%$ peptone, $0.3 \%$ yeast extract, $0.2 \% \mathrm{KH}_{2} \mathrm{PO}_{4}, 0.1 \% \mathrm{NaCl}, 0.01 \% \mathrm{MgSO}_{4} 7 \mathrm{H}_{2} \mathrm{O}$, and $1.8 \%$ Tween 80 (pH 5.5).

flavinogenic strain, being only $1 / 14$ th of that of the flavinogenic strain. The $\mathrm{pH}$ curve followed the same pattern as that of the flavinogenic strain. In conclusion, it was found that the nonflavinogenic strain has a slower growth rate and trace yields of the vitamin in comparison with the flavinogenic strain.

Next, the dynamic changes of the nucleotide pools in the nonflavinogenic strain under these cultural conditions were examined to detect substantial differences from the flavinogenic strain. The results are shown in Fig. 5, from which it can be seen that the general appearance of chromatograms in the nonflavinogenic 


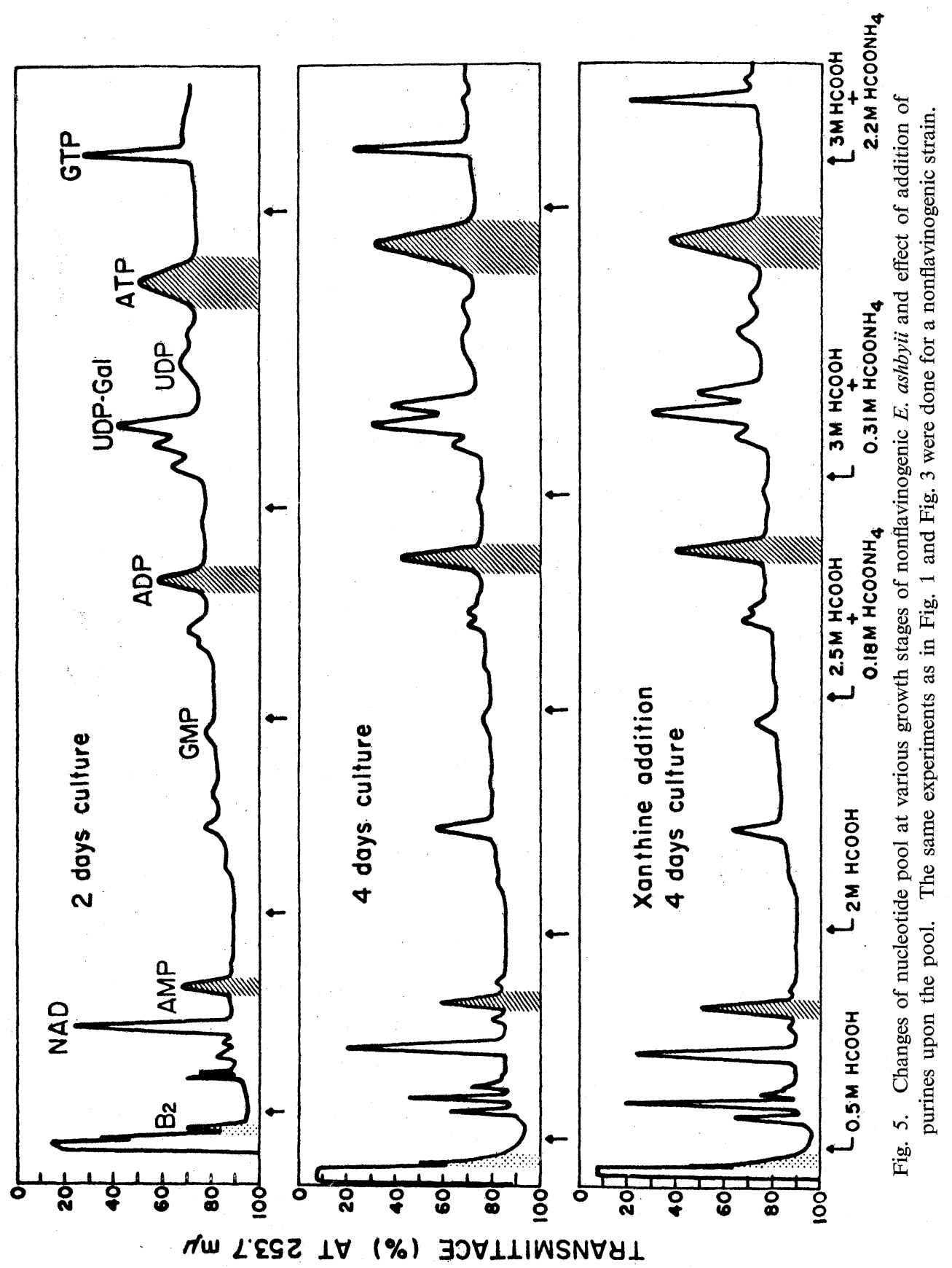


strain differed noticeably from those of the flavinogenic strain in that total nucleotide content of 4-day culture at the growth maximum is higher than that of 2-day culture at the exponential phase of growth. This indicates a good correspondence between the stage of maximum growth and the stage of largest nucleotide pools in the nonflavinogenic strain.

Table 3. Effect of xanthine on relative amounts of adenosine nucleotides in a nonflavinogenic strain of $E_{\text {. }}$ ashbyii. The relative amounts were calculated from Fig. 5 .

\begin{tabular}{lcccccc}
\hline & \multicolumn{2}{c}{ None (2 days) } & \multicolumn{2}{c}{ None (4 days) } & \multicolumn{2}{c}{ Xanthine (4 days) } \\
\hline NAD & 1 & - & 1 & - & 1 & - \\
& $\left(0.56^{\mathrm{a}}\right)$ & & $\left(0.66^{\mathrm{a}}\right)$ & & $\left(0.59^{\mathrm{a}}\right)$ & \\
AMP & 0.24 & 1 & 0.21 & 1 & 0.46 & 1 \\
ADP & 0.37 & 1.5 & 0.59 & 2.8 & 0.83 & 1.8 \\
ATP & 0.55 & 2.3 & 1.8 & 8.6 & 1.5 & 3.3 \\
AMP +ADP+ATP & 1.2 & - & 2.6 & - & 2.8 & - \\
\hline
\end{tabular}

a $\mu$ moles as AMP.

The relative amounts of the pools of adenosine nucleotides and NAD are given in Table 3, which indicates more clearly the difference in the nucleotide pool changes with cultivation times in the two strains.

Furthermore, the effects of exogenous purines on the nucleotide pools of the nonflavinogenic strain were examined at 4 days culture (growth maximum). However, as seen in Fig. 5, the noticeable effects of purines found at the growth maximum of 2 days culture in the flavinogenic strain were not observed.

Table 3 demonstrates in more detail that the addition of xanthine did not cause an increase of the ATP pool accompanied by a decrease of the AMP pool, as detected in the flavinogenic strain, but total adenosine nucleotide pools were almost unchanged.

\section{DISCUSSION}

It may be seen from the experiments described above that the stage of the maximal amounts of nucleotides coincides with the stage of maximal growth in the nonflavinogenic strain, and in the flavinogenic strain, the maximal pools of nucleotides were achieved about 1 day before the maximal growth stage. However, riboflavin production started simultaneously with the decrease of the nucleotide pools in the flavinogenic strain (Figs. 1,2). These results indicate that in the case of the flavinogenic strain, neither nucleotide synthesis nor riboflavin production are coupled to the growth, but the decrease of the nucleotide pool and the increase of riboflavin formation seem to be closely related. Since the maximum content of nucleotides is reached a considerable time before the maximum growth of this strongly flavinogenic strain, the mycelia are possibly preparing for rapid 
riboflavin production 2 or 3 days after cultivation.

On the other hand, it has been clarified in Candida utilis(18), Chlorella(19), and Bacillus(20) that the purines added to the cultural medium first enter into an intracellular purine pool and then into a nucleotide pool. Thus, the exogenous purines in the flavinogenic strain may proceed through the same metabolic pathway. Accordingly, exogenous purines would be converted to riboflavin through one of various nucleotides. This theory is supported by the physiological features of the flavinogenic strain and by the results of resting cell of E. ashbyii(21). Therefore, accumulation of some nucleotides might be expected on a column chromatogram. However, no noticeable accumulation of nucleotides or other purine derivatives was observed during the growth, as seen in Fig. 1. This may be attributed to the fact that this mold has the same rigid regulatory mechanism in its nucleotide pools as the above-mentioned species $(18-20)$ in spite of its high flavinogenic capacity. In fact, this is supported by the fact that the total content of adenosine nucleotide pools remains constant even after addition of purines (see Table 2).

However, when purines are added to the medium, a significant increase in the ATP fraction of the total adenosine nucleotide pools was observed in 2-day-old mycelia of the flavinogenic strain only. Since xanthine, which gave the highest riboflavin production $(21,23)$, brought about the most dynamic localization of ATP in the adenosine nucleotide pool (Table 2), it is quite conceivable that the ATP has a close association with flavinogenesis. This phenomenon raises the question of how the ATP is related to the riboflavin formation in this mold.

One simple answer is that ATP may act as a more effective precursor of riboflavin than any other purine derivative. According to this postulate, the addition of adenine into the medium should stimulate riboflavin production most effectively because exogenous adenine seems to be easily incorporated into the cell and converted to ATP in growing cells $(20,22)$. However, the stimulating effect of adenine is weaker than those of other purines $(21,23)$. Therefore, it is difficult to conclude that ATP acts as a precursor of riboflavin. Further evidence against this theory comes from the fact that GTP-not ATP-is the precursor involved in the biosynthetic pathway of toxoflavin(24) and various pteridines(2527), which have structures similar to lumazine, the most immediate precursor of riboflavin.

In view of the ingenious regulatory modes of ATP in nucleotide interconver$\operatorname{sion}(20,28-30)$, it is suggested that the localization of ATP in the adenosine nucleotide pools may play a role in the regulatory mechanism of the nucleotide pools closely related to the biosynthesis of riboflavin. WILSON(31) suggested that the rigid regulatory mechanism of a vitamin and its coenzyme synthesis might be attributed to the repression of the enzymes participating in the synthesis.

However, it is conceivable that the enzymes participating in riboflavin biosynthesis have already been synthesized 1 day after cultivation, as seen for riboflavin 
synthetase(32). Furthermore, the building blocks necessary for riboflavin synthesis, that is purine derivatives, have also been completely prepared after 1 day in the flavinogenic strain (Fig. 1 and Table 1). Thus, it is improbable that in this strain the enzymes in this pathway are synthesized explosively by depression at the phase of autolysis.

Accordingly, the authors propose that the synthesis of this vitamin and its coenzymes may be regulated by shifts of purine nucleotide pools in $E$. ashbyii. Another reason for this proposal is the fact that the reaction rate of GTP cyclohydrolase in the removal of $\mathrm{C}(8)$ from the purine, a reaction quite similar to the riboflavin biosynthetic mechanism, is not affected by various agents(33, 34).

If this view is accepted the question arises as to which pathways are specifically regulated by ATP. However, because ATP functions as a regulator of many pathways in purine nucleotide pools, further studies must be conducted in future.

\section{REFERENCES}

1) Schulman, M. D., Metabolic Pathway, ed. by Greenberg, D. M., Academic Press Inc., New York and London, Vol. II, p. 389 (1961).

2) Plaut, G. W. E., Metabolic Pathway, ed. by Greenberg, D. M., Academic Press Inc., New York and London, Vol. II, p. 673 (1961).

3) Goodwin, T. W., The Biosynthesis of Vitamins and Related Compounds, Academic Press Inc., New York and London, p. 24 (1963).

4) Plaut, G. W. E., Ann. Rev. Biochem., 30, 409 (1961).

5) Mitsuda, H. and Suzuki, Y., J. Vitaminol., 16, 172 (1970).

6) Hulbert, R. B., Schnitz, H., Brum, A. F., and Potter, V. R., J. Biol. Chem., 209, 23 (1954).

7) Burrows, S., Grylls, E. S. M., and Harrison, J. S., Nature, 170, 800 (1952).

8) Bock, R. M., Morell, S. A., Ling, N. S., and Lipton, S. H., Arch. Biochem. Biophys., 62, 253 (1956).

9) Brawerman, G., Hufugel, D. A., and Chargaff, E., Biochim. Biophys. Acta, 61, 340 (1962).

10) Vischer, E. and Chargaff, E., J. Biol. Chem., 176, 703 (1948).

11) Paladini, A. C. and Lelotr, L. F., Biochem. J., 51, 426 (1952).

12) Bacon, J. S. P. and Edelman, J., Biochem. J., 48, 114 (1951).

13) Jeans, A., Wise, C. S., and Dimer, R. J., Analytical Chem., 23, 415 (1951).

14) Jermyn, M. A. and Isherwood, F. A., Biochem. J., 44, 402 (1949).

15) Trevelyan, W. E., Procter, D. P., and Harrison, J. S., Nature, 166, 444 (1950).

16) Smith, J. D. and Markham, R. D., Biochem. J., 46, 509 (1950).

17) Mitsuda, H., Suzuki, Y., and Nakajima, K., Vitamins, 42, 380 (1970).

18) Covie, D. B. and Bolton, E. T., Biochim. Biophys. Acta, 25, 292 (1957).

19) Ammann, E. C. B. and LynCH, V. H., Biochim. Biophys. Acta, 87, 370 (1967).

20) Berlin, R. D. and Stadtman, E. R., J. Biol. Chem., 241, 2679 (1966).

21) Mitsuda, H. and NakaJima, K., J. Vitaminol., 18, 137 (1972).

22) Audley, B. G. and Goodwin, T. W., Biochim. J., 84, 587 (1962).

23) Mitsuda, H., Suzuki, Y., and Nakajima, K., Vitamins, 42, 386 (1970).

24) Levenberg, B. and Kaczmarek, D. K., Biochim. Biophys. Acta, 117, 272 (1966).

25) Guroff, G. and Strenkoski, C. A., J. Biol. Chem., 241, 2220 (1966).

26) Shiota, T. and Palumbo, M. D., J. Biol. Chem., 240, 4449 (1965).

27) Burg, A. W. and Brown, G. M., Biochim. Biophys. Acta, 117, 275 (1966). 
28) Mager, J. and Magasanik, B., J. Biol. Chem., 235, 1474 (1960).

29) Wyngaarden, J. B. and Ashton, D. M., J. Biol. Chem., 234, 1492 (1959).

30) Setlow, B., Burger, R., and Lowenstein, J. M., J. Biol. Chem., 241, 1244 (1966).

31) Wilson, A. C. and Pardee, A. B., J. Gen. Microbiol., 28, 283 (1962).

32) Suzuki, Y. and Mitsuda, H., Biochim. Biophys. Acta, 242, 500 (1971).

33) Burg, A. W. and Brown, G. M., J. Biol. Chem., 243, 2349 (1968).

34) Cone, J. and Guroff, G., J. Biol. Chem., 249, 979 (1971). 